\title{
Research Article New Classes of Charged Spheroidal Models
}

\author{
S. Thirukkanesh \\ Department of Mathematics, Eastern University, 30350 Chenkalady, Sri Lanka \\ Correspondence should be addressed to S. Thirukkanesh; thirukkanesh@yahoo.co.uk
}

Received 25 March 2013; Accepted 25 August 2013

Academic Editors: M. S. Dimitrijevic, A. Mesinger, L. Nicastro, A. S. Pozanenko, and A. Pradhan

Copyright (C) 2013 S. Thirukkanesh. This is an open access article distributed under the Creative Commons Attribution License, which permits unrestricted use, distribution, and reproduction in any medium, provided the original work is properly cited.

\begin{abstract}
New classes of exact solutions to the Einstein-Maxwell system is found in closed form by assuming that the hypersurface $t=$ constant is spheroidal. This is achieved by choosing a particular form for the electric field intensity. A class of solution is found for all positive spheroidal parameter $K$ for a specific form of electric field intensity. In general, the condition of pressure isotropy reduces to a difference equation with variable, rational coefficients that can be solved. Consequently, an explicit solution in series form is found. By placing restrictions on the parameters, it is shown that the series terminates and there exist two classes of solutions in terms of elementary functions. These solutions contain the models found previously in the limit of vanishing charge. Solutions found are directly relating the spheroidal parameter and electric field intensity. Masses obtained are consistent with the previously reported experimental and theoretical studies describing strange stars. A physical analysis indicates that these models may be used to describe a charged sphere.
\end{abstract}

\section{Introduction}

In recent years, there have been several investigations into the Einstein-Maxwell system of equations for static spherically symmetric gravitational fields with isotropic pressures in the presence of the electromagnetic field. In such study, regular interior spacetime is matched smoothly at the pressure free interface to the Reissner-Nordstrom exterior model. The models generated are useful to describe charged relativistic bodies with strong gravitational fields such as neutron stars. Gravitational collapse of a spherically symmetric distribution of matter to a point singularity may be avoided in the presence of electromagnetic field. In this situation, the gravitational attraction is counterbalanced by the repulsive Columbian force with the pressure gradient and, hence, charged fluids have a tendency to resist the gravitational collapse. This property persuades the researchers to work on charged perfect fluid distribution. Bonnor [1] has shown that charged dust solutions are expected to form a point like model of electron when its radius shrinks to zero. The presence of electromagnetic field affects the value of redshifts, luminosities, and maximum mass of a compact relativistic object (Ivanov [2], Sharma et al. [3]). Many exact solutions which satisfy the conditions for a physically acceptable charged relativistic sphere have been given by Ivanov [2], Thirukkanesh and Maharaj [4], and Gupta and Maurya [5], among others. Detailed studies of Sharma et al. [6] in cold compact objects, Sharma and Mukherjee [7] analysis of strange matter and binary pulsars and Sharma and Mukherjee [8] analysis of qark-diquark mixtures in equilibrium are of interest physically. Thomas et al. [9], Tikekar and Thomas [10], and Paul and Tikekar [11] have shown that charged relativistic matter is relevant in modeling core-envelope stellar system in which the stellar core is an isotropic fluid surrounded by a layer of anisotropic fluid.

Vaidya and Tikekar [12] proposed the geometry of the spacelike hypersurfaces generated by $t=$ constant are of 3 -spheroid to generate exact solutions since it provides a clear geometrical interpretation: the models with spheroidal geometries directly related to the physical situations. Tikekar [13] found an exact solution for a particular spheroidal geometry which could be used to model superdense neutron stars of densities in the range of $10^{14} \mathrm{gcm}^{-3}$; this solution has been generalized by Maharaj and Leach [14]. There have been extensive studies on charged spheroidal stars by considering a particular form for the electric field in recent years [15-23]. These charged spheroidal models contain uncharged neutron stars in the relevant limit and are consequently relevant in 
the description of dense astrophysical objects. Therefore, the study of charged fluid spheres in static spherically symmetric spacetimes is important in relativistic astrophysics.

The objective of this paper is to generate new classes of charged spheroidal solutions in terms of elementary function, which may be used to describe the interior of a relativistic compact sphere. In Section 2, the Einstein-Maxwell system of equations is expressed for static spherically symmetric spacetime. In Section 3, particular forms for one of the gravitational potentials with spheroidal parameter and the electric field intensity are chosen, which reduces the condition of pressure isotropy to a second order linear differential equation in the remaining gravitational potential. In Section 4, a class of solutions for a particular parameter value is first obtained. In general, the solution is obtained in series form using the method of Frobenius, and then two categories of solutions in terms of elementarily functions are derived by placing restrictions on the parameters. The physical features are illustrated graphically, and numerical values of some physical quantities are calculated for a particular example in Section 5 .

\section{Field Equations}

The gravitational field should be static and spherically symmetric to describe the internal structure of a charged dense compact relativistic sphere. Therefore, the generic form of the line element for describing such configuration is given by

$$
d s^{2}=-e^{2 v(r)} d t^{2}+e^{2 \lambda(r)} d r^{2}+r^{2}\left(d \theta^{2}+\sin ^{2} \theta d \phi^{2}\right)
$$

in Schwarzschild coordinates $\left(x^{a}\right)=(t, r, \theta, \phi)$, where $v(r)$ and $\lambda(r)$ are arbitrary function of radial coordinate $r$. The Einstein-Maxwell system of field equations, for the metric (1), can be written in the form

$$
\begin{aligned}
& \frac{1}{r^{2}}\left(1-e^{-2 \lambda}\right)+\frac{2 \lambda^{\prime}}{r} e^{-2 \lambda}=\rho+\frac{1}{2} E^{2}, \\
& \frac{-1}{r^{2}}\left(1-e^{-2 \lambda}\right)+\frac{2 \nu^{\prime}}{r} e^{-2 \lambda}=p-\frac{1}{2} E^{2}, \\
& e^{-2 \lambda}\left(\nu^{\prime \prime}+\nu^{\prime 2}+\frac{\nu^{\prime}}{r}-v^{\prime} \lambda^{\prime}-\frac{\lambda^{\prime}}{r}\right)=p+\frac{1}{2} E^{2}, \\
& \sigma=\frac{1}{r^{2}} e^{-\lambda}\left(r^{2} E\right)^{\prime} \text {. }
\end{aligned}
$$

The energy density $\rho$ and the pressure $p$ are measured relative to the commoving fluid 4-velocity $u^{a}=e^{-v} \delta_{0}^{a}$ and primes denote differentiation with respect to the radial coordinate $r$. The quantities $E$ and $\sigma$ denote the electric field intensity and the proper charge density, respectively. In the system (2a) $-(2 \mathrm{~d})$, the units used are such that the coupling constant $8 \pi G / c^{4}=1$ and the speed of light $c=1$. This system of equations determines the behaviour of the gravitational field for a charged perfect fluid source. When $E=0$ the EinsteinMaxwell system (2a)-(2d) reduces to the uncharged Einstein system.

\section{Choosing Gravitational Potential and Electric Field Intensity}

The aim is to seek solutions to the Einstein-Maxwell system (2a) $-(2 \mathrm{~d})$ by making explicit choices for the gravitational potential $e^{2 \lambda(r)}$ and electric field intensity $E$ on physical grounds. The system $(2 \mathrm{a})-(2 \mathrm{~d})$ comprises four equation with six unknowns $\lambda, \nu, \rho, p, E$, and $\sigma$ so that it is necessary to choose two of the variables to integrate the system. In this treatment, $\lambda$ and $E$ are specified. A particular choice for $\lambda$ is made such that

$$
e^{2 \lambda(r)}=\frac{1-K r^{2} / R^{2}}{1-r^{2} / R^{2}},
$$

where $K$ and $R$ are real constants. The above form has been used previously by Tikekar [13] and Maharaj and Leach [14] to study the behaviour of uncharged superdense stars. Note that the choice (3) for the gravitational potential $\lambda$ restricts the geometry of the 3-dimensional hypersurfaces $t=$ constant to be spheroidal for $K \neq 0$ and spherical for $K=0$.

Eliminating $p$ from (2b) and (2c), for the particular form (3), one obtain the condition of pressure isotropy:

$$
\begin{aligned}
\left(1-\frac{K r^{2}}{R^{2}}\right)^{2} E^{2}= & \left(1-\frac{K r^{2}}{R^{2}}\right)\left(1-\frac{r^{2}}{R^{2}}\right)\left(\nu^{\prime \prime}+\nu^{\prime 2}-\frac{\nu^{\prime}}{r}\right) \\
& -(1-K)\left(\frac{r}{R^{2}}\right)\left(v^{\prime}+\frac{1}{r}\right) \\
& +\frac{1-K}{R^{2}}\left(1-\frac{K r^{2}}{R^{2}}\right) .
\end{aligned}
$$

The transformation

$$
\psi(x)=e^{\nu(r)}, \quad x^{2}=1-\frac{r^{2}}{R^{2}}
$$

reduces the condition (4) for pressure isotropy to a convenient form

$$
\begin{aligned}
& \left(1-K+K x^{2}\right) \ddot{\psi}-K x \dot{\psi} \\
& \quad+\left(\frac{\left(1-K+K x^{2}\right)^{2} R^{2} E^{2}}{x^{2}-1}+K(K-1)\right) \psi=0
\end{aligned}
$$

in terms of the new variables $\psi$ and $x$, where dots denote differentiation with respect to $x$.

In terms of new variable $x$, the Einstein-Maxwell system (2a)-(2d) becomes

$$
\begin{gathered}
\rho=\frac{1-K}{R^{2}} \frac{\left(3-K+K x^{2}\right)}{\left(1-K+K x^{2}\right)^{2}}-\frac{1}{2} E^{2}, \\
p=\frac{1}{R^{2}\left(1-K+K x^{2}\right)}\left(-2 x \frac{\dot{\psi}}{\psi}+K-1\right)+\frac{1}{2} E^{2}, \\
\sigma^{2}=\frac{\left[2 x E-\left(1-x^{2}\right) \dot{E}\right]^{2}}{R^{2}\left(1-x^{2}\right)\left(1-K+K x^{2}\right)}
\end{gathered}
$$


for the choice (3). Note that in (7a)-(7c), $\rho, p$, and $\sigma$ are defined in terms of $E$. Equation (6) may be integrable if a particular choice of the electric field intensity $E$ is made. For mathematical convenient one may take

$$
\begin{aligned}
& E^{2}=\frac{2 \beta K x\left(1-x^{2}\right)}{\left(1-K+K x^{2}\right)^{2} R^{2}} \frac{\dot{\psi}}{\psi}, \\
& \text { that is, } E^{2}=\frac{\beta K r}{\left(r^{2}-R^{2}\right)} \frac{\nu^{\prime}}{e^{4 \lambda}},
\end{aligned}
$$

where $\beta$ is a constant which is different from the choice of Komathiraj and Maharaj [15]. On substituting (8) into (6), we obtain a second order linear differential equation

$$
\left(1-K+K x^{2}\right) \ddot{\psi}-K(1+2 \beta) x \dot{\psi}+K(K-1) \psi=0
$$

in $\psi$. It is expected that investigation of (9) will produce physically reasonable models of charged stars since $\beta=0$ yields models found previously by Maharaj and Leach [14] for neutron stars which contain Tikekar [13] superdense stars as special case.

\section{Solution}

It is clear that the solution of the Einstein-Maxwell system depends on the integrability of (9). One may consider the following two cases.

4.1. Particular Case. When $\beta=-1$, (9) becomes

$$
\left(1-K+K x^{2}\right) \ddot{\psi}+K x \dot{\psi}+K(K-1) \psi=0 .
$$

If we utilize the transformation

$$
Z=\frac{1}{\sqrt{K}} \ln \left[K x+\sqrt{K^{2} x^{2}+K(1-K)}\right]
$$

equation (10) reduces to the equation of free oscillation

$$
\frac{d^{2} \psi}{d Z^{2}}+K(K-1) \psi=0,
$$

for $K>0$. The solutions of (12) become

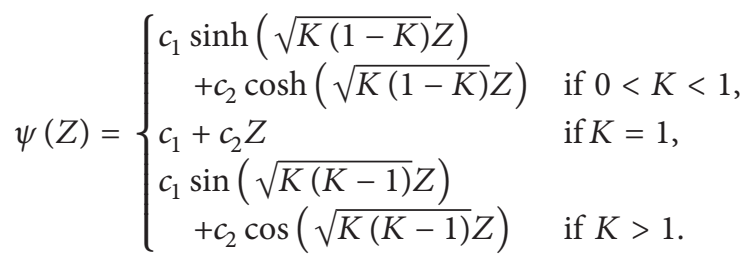

This becomes

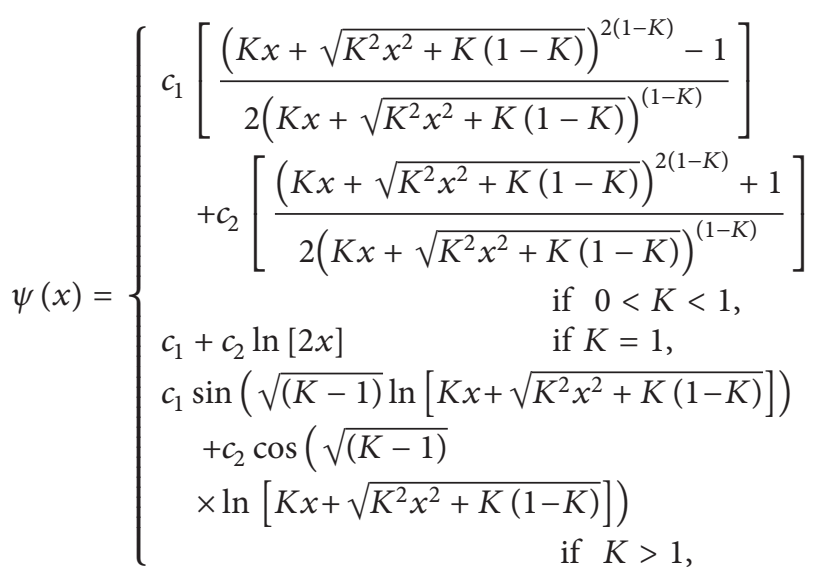

in terms of the variable $x$. Thus, a class of exact solution to the Einstein-Maxwell system is generated for all positive value of $K$. Solution (14) is given in simple form which is an advantage for physical analysis.

4.2. General Case. Note that (9) can be transformed to a hypergeometric equation. However, it is impossible to express the solutions in terms of elementary function for all $K$. In general, the solution will be given in terms of special functions. Solutions in a simple form are important for a detailed physical analysis. Hence, first I attempt to obtain a general solution of (9) in a series form using the method of Frobenius and then demonstrate the possibility to extract solutions in terms of polynomials and algebraic functions by imposing restrictions on the parameters.

4.2.1. Series Solution. Since $x=0$ is a regular point of the differential equation (9), we can apply the method of Frobenius about $x=0$ to obtain a series solution. Thus, we assume that

$$
\psi(x)=\sum_{i=0}^{\infty} a_{i} x^{i}
$$

is a solution of (9). To express the solution, the coefficients of the series $a_{i}$ need to determined explicitly. Substituting (15) into (9) we obtain

$$
\begin{aligned}
& 2(1-K) a_{2}+K(K-1) a_{0} \\
& +\left[6(1-K) a_{3}+K(K-2-2 \beta) a_{1}\right] x \\
& +\sum_{i=2}^{\infty}\left\{(1-K)(i+1)(i+2) a_{i+2}\right. \\
& \left.\quad+K[K-1+i(i-2-2 \beta)] a_{i}\right\} x^{i}=0 .
\end{aligned}
$$


For the validity of (16), we must have

$$
\begin{gathered}
2(1-K) a_{2}+K(K-1) a_{0}=0, \\
6(1-K) a_{3}+K(K-2-2 \beta) a_{1}=0, \\
(1-K)(i+1)(i+2) a_{i+2} \\
+K[K-1+i(i-2-2 \beta)] a_{i}=0, \quad i \geq 2 .
\end{gathered}
$$

It remains to obtain the coefficients $a_{i}$ from the system (17a)$(17 c)$. Equation (17c) is the linear difference equation governing the structure of the solution. The difference equation $(17 \mathrm{c})$ consists of variable, rational coefficients.

First consider the even coefficients $a_{0}, a_{2}, a_{4}, \ldots$ These coefficients generate a pattern

$$
a_{2 i}=\left(\frac{K}{K-1}\right)^{i} \frac{1}{(2 i) !} \prod_{q=1}^{i}[K-1+(2 q-2)(2 q-4-2 \beta)] a_{0}
$$

where the symbol $\prod$ denotes multiplication. The odd coefficients $a_{1}, a_{3}, a_{5}, \ldots$ can be written in the form

$$
\begin{aligned}
a_{2 i+1}= & \left(\frac{K}{K-1}\right)^{i} \frac{1}{(2 i+1) !} \\
& \times \prod_{q=1}^{i}[K-1+(2 q-1)(2 q-3-2 \beta)] a_{1} .
\end{aligned}
$$

Hence, the difference equation (17c) has been solved and all nonzero coefficients are expressible in terms of the leading coefficients $a_{0}$ and $a_{1}$. Thus, from (15), (18), and (19) the solution of (9) becomes

$$
\begin{aligned}
\psi(x)=a_{0}(1 & +\sum_{i=1}^{\infty}\left(\frac{K}{K-1}\right)^{i} \frac{1}{(2 i) !} \\
& \left.\times \prod_{q=1}^{i}[K-1+4(q-1)(q-2-2 \beta)] x^{2 i}\right) \\
& +a_{1}\left(x+\sum_{i=1}^{\infty}\left(\frac{K}{K-1}\right)^{i} \frac{1}{(2 i+1) !}\right. \\
& \left.\times \prod_{q=1}^{i}[K-1+(2 q-1)(2 q-3-2 \beta)] x^{2 i+1}\right),
\end{aligned}
$$

where $a_{0}$ and $a_{1}$ are arbitrary constants. Therefore, the general solution of (9) for the choice (15) is given by

$$
\psi(x)=a_{0} \psi_{1}(x)+a_{1} \psi_{2}(x)
$$

where

$$
\begin{aligned}
\psi_{1}(x)= & \left(1+\sum_{i=1}^{\infty}\left(\frac{K}{K-1}\right)^{i} \frac{1}{(2 i) !}\right. \\
& \left.\times \prod_{q=1}^{i}[K-1+4(q-1)(q-2-2 \beta)] x^{2 i}\right), \\
\psi_{2}(x)= & \left(x+\sum_{i=1}^{\infty}\left(\frac{K}{K-1}\right)^{i} \frac{1}{(2 i+1) !} \prod_{q=1}^{i}\right. \\
& \left.\times[K-1+(2 q-1)(2 q-3-2 \beta)] x^{2 i+1}\right)
\end{aligned}
$$

are linearly independent solutions of (9).

It is interesting to observe that when $K=0$ the series solution (21) reduces to a simple form

$$
\psi(x)=a_{0}+a_{1} x .
$$

In this case, the electric field intensity $E$ vanishes and there is no charge.

Note that when $a_{0}=0$ and $a_{1}=1$, the line element (1) takes the form

$$
\begin{aligned}
d s^{2}= & -\left(1-\frac{r^{2}}{R^{2}}\right) d t^{2}+\left(1-\frac{r^{2}}{R^{2}}\right)^{-1} d r^{2} \\
& +r^{2}\left(d \theta^{2}+\sin ^{2} \theta d \phi^{2}\right) .
\end{aligned}
$$

The above metric corresponds to the familiar isotropic uncharged de Sitter model. form

When $a_{1}=0$, the line element (1) takes the particular

$$
d s^{2}=-a_{0}^{2} d t^{2}+\left(1-\frac{r^{2}}{R^{2}}\right)^{-1} d r^{2}+r^{2}\left(d \theta^{2}+\sin ^{2} \theta d \phi^{2}\right) .
$$

The above metric corresponds to the well-known isotropic uncharged Einstein model.

4.2.2. Terminating Series. The general solution (21) can be expressed in terms of polynomial and algebraic function for restricted values of the parameter $K$. This will happen because series (22a) and (22b) terminate for restricted values of $K$. Using this feature, it is possible to generate two sets of solutions in terms of elementary functions by determining specific restrictions on $K$, as demonstrated below. For simplicity, the difference equation (17c) is used instead of the series (22a) and (22b) to obtain the solutions in terms of elementary functions.

First Solution. Firstly consider the polynomials of even degree. If we set

$$
\begin{gathered}
i=2(j-1), \\
K=2-(2 n-1)^{2}+4 n \beta
\end{gathered}
$$


then for a fixed integer $n>1,(17 \mathrm{c})$ become

$$
a_{2 j}=-\gamma \frac{(n-\beta+j-2)(n-j+1)}{2 j(2 j-1)} a_{2 j-2},
$$

where $\gamma=4-1 /[n(n-1-\beta)]$. Observe that (27) implies $a_{2(n+1)}=0$. It is easy to see that the remaining coefficients $a_{2(n+2)}, a_{2(n+3)}, a_{2(n+4)}, \ldots$ vanish and (27) has the solution

$$
a_{2 j}=(-\gamma)^{j} \frac{(n-\beta+j-2) !(n-1) !}{(2 j) !(n-\beta-1) !(n-j) !}, \quad 0 \leq j \leq n,
$$

where I have set $a_{0}=1 / n(n-\beta-1)$. Therefore, from (15) and, (28), we can express the polynomial in even powers of $x$ as

$$
f_{1}(x)=\sum_{j=0}^{n}(-\gamma)^{j} \frac{(n-\beta+j-2) !(n-1) !}{(2 j) !(n-\beta-1) !(n-j) !} x^{2 j}
$$

for $K=2-(2 n-1)^{2}+4 n \beta$.

Secondly, consider the polynomials of odd degree. For this case, if we set

$$
\begin{gathered}
i=2(j-1)+1, \\
K=2\left[1-2 n^{2}+(2 n+1) \beta\right]
\end{gathered}
$$

then for a fixed integer $n>0,(17 \mathrm{c})$ becomes

$$
a_{2 j+1}=-\mu \frac{(n+j-1-\beta)(n-j+1)}{2 j(2 j+1)} a_{2 j-1},
$$

where $\mu=4-4 /[(2 n+1)(2 n-1-2 \beta)]$. Observe that (31) implies $a_{2(n+1)+1}=0$. Consequently, the remaining coefficients $a_{2(n+2)+1}, a_{2(n+3)+1}, a_{2(n+4)+1}, \ldots$ vanish and (31) has the solution

$$
a_{2 j+1}=(-\mu)^{j} \frac{(n-\beta+j-1) !(n-1) !}{(2 j+1) !(n-\beta-1) !(n-j) !}, \quad 0 \leq j \leq n,
$$

where I have set $a_{1}=1 / n$. Therefore, from (15) and (32), we can express the polynomial in odd powers of $x$ as

$$
g_{1}(x)=\sum_{j=0}^{n}(-\mu)^{j} \frac{(n-\beta+j-1) !(n-1) !}{(2 j+1) !(n-\beta-1) !(n-j) !} x^{2 j+1}
$$

for $K=2\left[1-2 n^{2}+(2 n+1) \beta\right]$.

Polynomial (29) and (33) comprise the first solution of (9) for appropriate values of $K$.

Second Solution. Assume the second solution of (9) to be of the form

$$
\psi(x)=u(x)\left(1-K+K x^{2}\right)^{(3 / 2)+\beta},
$$

where $u(x)$ is an arbitrary function. Substituting $\psi$ in (9) we obtain

$$
\begin{aligned}
& \left(1-K+K x^{2}\right) \frac{d^{2} u}{d x^{2}}+K(5+2 \beta) x \frac{d u}{d x} \\
& +K(K+2+2 \beta) u=0,
\end{aligned}
$$

which is a linear differential equation in $u(x)$.
Observe that (9) and (35) are of the same type. As in Section 4.2.1, we can first find a general series solution and then two classes of polynomial solution (in even powers of $x$ and in odd powers of $x$ ) for (35) using the above technique. Hence, I present the final form of the solution: the polynomial in even powers of $x$ leads to the expression

$$
u(x)=\sum_{j=0}^{n-\beta-1}(-\mu)^{j} \frac{(n+j) !(n-\beta-1) !}{(2 j) !(n-1) !(n-\beta-j-1) !} x^{2 j}
$$

for $K=2\left[1-2 n^{2}+(2 n+1) \beta\right]$, where the real constant $\beta$ is restricted as integer such that $\beta \leq n-1$; the polynomial in odd powers of $x$ leads to the result

$$
u(x)=\sum_{j=0}^{n-\beta-2}(-\gamma)^{j} \frac{(n+j) !(n-\beta-2) !}{(2 j+1) !(n-2) !(n-\beta-j-2) !} x^{2 j+1}
$$

for $K=2-(2 n-1)^{2}+4 n \beta$, where the real constant $\beta$ is restricted as integer such that $\beta \leq n-2$ in this case.

Hence, the solutions to (9) becomes:

$$
\begin{aligned}
g_{2}(x)= & \left(1-K+K x^{2}\right)^{(3 / 2)+\beta} \\
& \times \sum_{j=0}^{n-\beta-1}(-\mu)^{j} \frac{(n+j) !(n-\beta-1) !}{(2 j) !(n-1) !(n-\beta-j-1) !} x^{2 j}
\end{aligned}
$$

for $K=2\left[1-2 n^{2}+(2 n+1) \beta\right]$, where $\beta$ is an integer such that $\beta \leq n-1$;

$$
\begin{aligned}
f_{2}(x)= & \left(1-K+K x^{2}\right)^{(3 / 2)+\beta} \\
& \times \sum_{j=0}^{n-\beta-2}(-\gamma)^{j} \frac{(n+j) !(n-\beta-2) !}{(2 j+1) !(n-2) !(n-\beta-j-2) !} x^{2 j+1}
\end{aligned}
$$

for $K=2-(2 n-1)^{2}+4 n \beta$, where $\beta$ is an integer such that $\beta \leq n-2$.

The algebraic functions (38) and (39) comprise the second solution of (9) for appropriate values of $K$.

Exact Solution. The solutions generated in Section 4.2.2 can be expressed in terms of two classes of elementary functions. The first category of solution for $\psi(x)=f(x)$ is

$$
\begin{aligned}
f(x)= & A f_{1}(x)+B f_{2}(x) \\
= & A \sum_{j=0}^{n}(-\gamma)^{j} \frac{(n-\beta+j-2) !(n-1) !}{(2 j) !(n-\beta-1) !(n-j) !} x^{2 j} \\
& +B\left(1-K+K x^{2}\right)^{(3 / 2)+\beta} \\
& \times \sum_{j=0}^{n-\beta-2}(-\gamma)^{j} \frac{(n+j) !(n-\beta-2) !}{(2 j+1) !(n-2) !(n-\beta-j-2) !} x^{2 j+1}
\end{aligned}
$$


for the values

$$
\begin{aligned}
& \gamma=4-\frac{1}{n(n-1-\beta)}, \\
& K=2-(2 n-1)^{2}+4 n \beta, \quad \text { where } \beta \leq n-2 .
\end{aligned}
$$

The second category of solution for $\psi(x)=g(x)$ is

$$
\begin{aligned}
g(x)= & A g_{1}(x)+B g_{2}(x) \\
= & A \sum_{j=0}^{n}(-\mu)^{j} \frac{(n-\beta+j-1) !(n-1) !}{(2 j+1) !(n-\beta-1) !(n-j) !} x^{2 j+1} \\
& +B\left(1-K+K x^{2}\right)^{(3 / 2)+\beta} \\
& \times \sum_{j=0}^{n-\beta-1}(-\mu)^{j} \frac{(n+j) !(n-\beta-1) !}{(2 j) !(n-1) !(n-\beta-j-1) !} x^{2 j}
\end{aligned}
$$

for the values

$$
\begin{aligned}
\mu & =4-\frac{4}{(2 n+1)(2 n-1-2 \beta)}, \\
K & =2\left[1-2 n^{2}+(2 n+1) \beta\right], \quad \text { where } \beta \leq n-1 .
\end{aligned}
$$

In (40) and (42), $A$ and $B$ are arbitrary constants.

The solutions (40) and (42) are given completely in terms of elementary functions: this has the advantage of facilitating the analysis of physical feature of the stellar interior. These solutions are applicable to a charged superdense star with spheroidal geometry. Note that this treatment has combined both charged and neutral cases for a relativistic star: by setting $\beta=0$ one obtain the solution for neutral case directly.

From these general class of solutions (40) and (42), it is possible to regain particular solutions found in the past. For example, the solutions (40) and (42) reduce to the uncharged models of Maharaj and Leach [14] when $\beta=0$ which contain the Tikekar [13] superdense neutron star model for $K=-7$. Other explicit functional forms for $\psi$ are obtainable which could be useful to study dense stars. For example, if we set $\beta=1$ and $K=-4(n=2)$ then (42) becomes

$$
\psi=A x\left[\frac{1}{2}-\frac{8}{15} x^{2}+\frac{64}{375} x^{4}\right]+2 B\left(5-4 x^{2}\right)^{5 / 2} .
$$

For this case, the line element takes a simple form

$$
\begin{aligned}
d s^{2}=- & {\left[\frac{A}{750} \sqrt{1-\frac{r^{2}}{R^{2}}}\right.} \\
& \times\left[103+144 \frac{r^{2}}{R^{2}}+128 \frac{r^{4}}{R^{4}}\right] \\
& \left.+2 B\left(1+4 \frac{r^{2}}{R^{2}}\right)\right]^{2} d t^{2}+\frac{1+4 r^{2} / R^{2}}{1-r^{2} / R^{2}} d r^{2} \\
+ & r^{2}\left(d \theta^{2}+\sin ^{2} \theta d \phi^{2}\right),
\end{aligned}
$$

in terms of the original variable $r$.

\section{Physical Analysis}

It is easy to observe from the solutions (40) and (42) that the spheroidal parameter increases with $\beta$ so that the electric field intensity affect the spheroidal parameter. For vanishing of pressure at the boundary $r=b$ in the solutions (14), (40), and (42), we require $p(b)=0$, that gives the condition

$$
\begin{aligned}
& \sqrt{1-\frac{b^{2}}{R^{2}}\left(\frac{(\beta+2) K\left(b^{2} / R^{2}\right)}{\left[1-K\left(b^{2} / R^{2}\right)\right]}\right)} \\
& \times\left[\frac{\dot{\psi}}{\psi}\right]_{x=\sqrt{1-\left(b^{2} / R^{2}\right)}}+K-1=0 .
\end{aligned}
$$

The generated interior metric matches smoothly with the exterior Reissnar-Nordstrom metric:

$$
\begin{aligned}
d s^{2}= & -\left(1-\frac{2 m}{r}+\frac{q^{2}}{r^{2}}\right) d t^{2}+\left(1-\frac{2 m}{r}+\frac{q^{2}}{r^{2}}\right)^{-1} d r^{2} \\
& +r^{2}\left(d \theta^{2}+\sin ^{2} \theta d \phi^{2}\right)
\end{aligned}
$$

across the boundary $r=b$, where $m$ and $q$ are the total mass and charge of the sphere. This generate the relationship between $b, \beta, K, R$ and the arbitrary constants involved in the solutions $\psi$ as follows:

$$
\begin{aligned}
& \left(1-\frac{2 m}{r}+\frac{q^{2}}{r^{2}}\right)=\left[\psi^{2}\right]_{x=\sqrt{1-\left(b^{2} / R^{2}\right)}} \\
& \left(1-\frac{2 m}{r}+\frac{q^{2}}{r^{2}}\right)^{-1}=\frac{1-K\left(b^{2} / R^{2}\right)}{1-\left(b^{2} / R^{2}\right)} .
\end{aligned}
$$

The conditions (46) and (48a)-(48b) place the restrictions on the parameters involved in these equations. However, there are sufficient free parameters to satisfy the necessary condition that arise for the model under investigation.

Now, we shall show that the obtained models are physically reasonable by plotting for a particular solution (45) as illustrated in Figures 1-7 by assuming the parameter values $A=-10, B=-1$, and $R=16 \mathrm{~km}$ in the interval $0 \leq r \leq 1$, where $r$ is in $\mathrm{km}$. The software package Mathematica was utilized to generate the plots for $e^{2 \nu}, e^{2 \lambda}, \rho, p, E^{2}, d p / d \rho$, and $p$ versus $\rho$. Gravitational potentials are plotted in Figures 1 and 2 which are nonsingular at the center and increasing from center to the boundary. The behaviour of the energy density is plotted in Figure 3, which is positive and monotonically decreasing towards the boundary of the stellar object. The behaviour of matter pressure $p$ is plotted in Figure 4, which is regular, monotonically decreasing throughout the stellar interior. The behaviour of the electric field intensity is described in Figure 5, which is continuous and well behaved. The derivative $d p / d \rho$ is plotted in Figure 6, which is $0 \leq$ $d p / d \rho \leq 1$ throughout the interior of the stellar object. Therefore, the speed of the sound is less than the speed of the light and causality is maintained. The pressure $p$ verses the density $\rho$ is plotted in Figure 7, and this looks like a straight line so that the equation of state approximate to a linear 


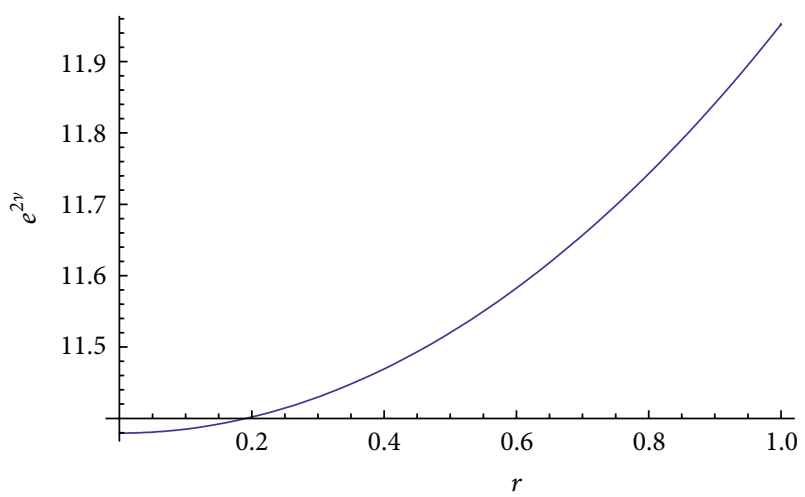

FIGURE 1: Gravitational potential $e^{2 \nu}$.

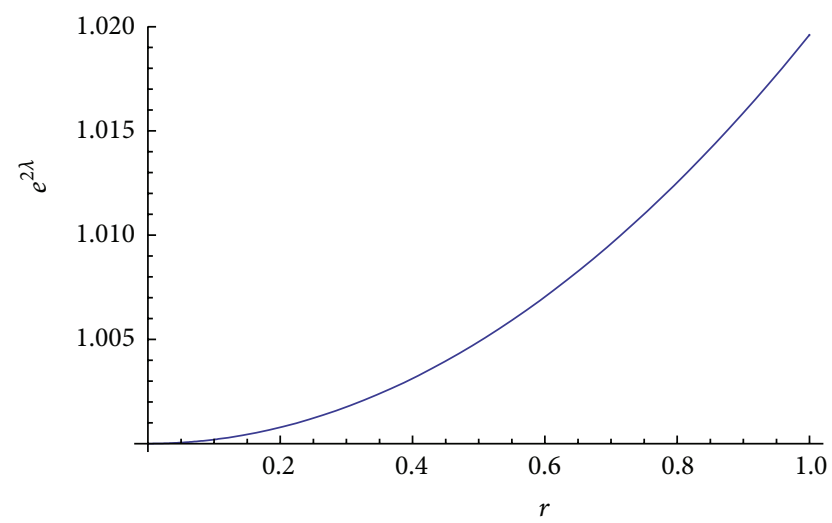

Figure 2: Gravitational potential $e^{2 \lambda}$.

relationship between $p$ and $\rho$. Thus, we have demonstrated that the particular solution satisfies the requirements for a physically reasonable stellar interior in the context of general relativity.

Numerical values for the physical quantities for the metric (45) for various radius are given in Table 1, where the constant of integration $A=0$ is assumed for simplicity. The radius of the star $b$ is chosen to be compared with experimental values reported for realistic stars. In all cases, it is noted that at the surface the pressure $p(b)=0$ (up to 8 decimal places), the density $\rho(b)>0$ and the charge $q(b)>0$; at the centre the pressure $p(0)>0$ and the density $\rho(0)>0$. The mass of the sphere of radius $7.07 \mathrm{~km}$ obtained in Table 1 is comparable with the experimentally determined value $1.44 M_{\odot}$ of the strange star SAX J1808.43658(SS1) having the same radius [24]. Moreover, similar value of mass was theoretically obtained for SAX J1808.43658(SS1) by Dey et al. [25], Sharma and Maharaj [26] and Tikekar and Jotania [27]. A similar mass value for the SAX J1808.4-3658(SS2) was reported by Tikekar and Jotania [27]. Experimental observations suggest a star model for Her. X1 , which is estimated to have a mass between 1.1-1.8 $M_{\odot}$ and the radius between $6-7.7 \mathrm{~km}$ [28]. It is noted that the mass obtained for radius $7.7 \mathrm{~km}$ and $R=9.445343 \mathrm{~km}$ in Table 1 corroborates with this experimentally estimated result for Her. X-1. The mass obtained for $4 \mathrm{U} 1820-30$ is

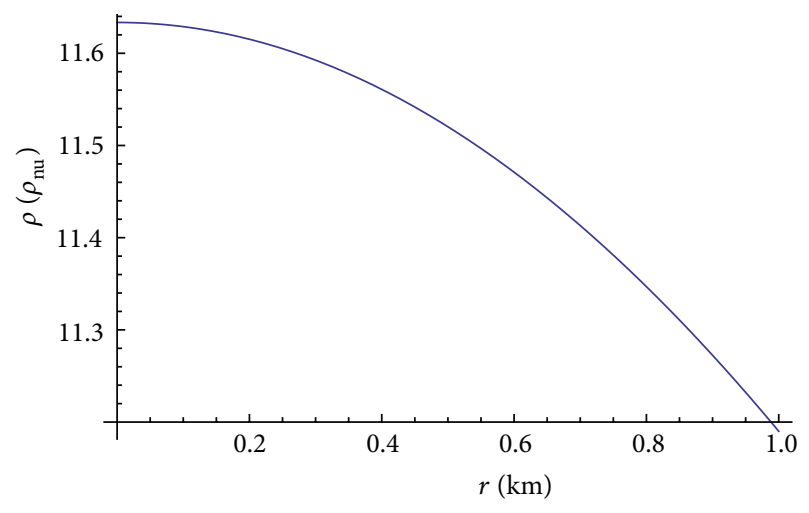

FIGURE 3: Energy density in terms of nuclear density $\rho_{n u}$.

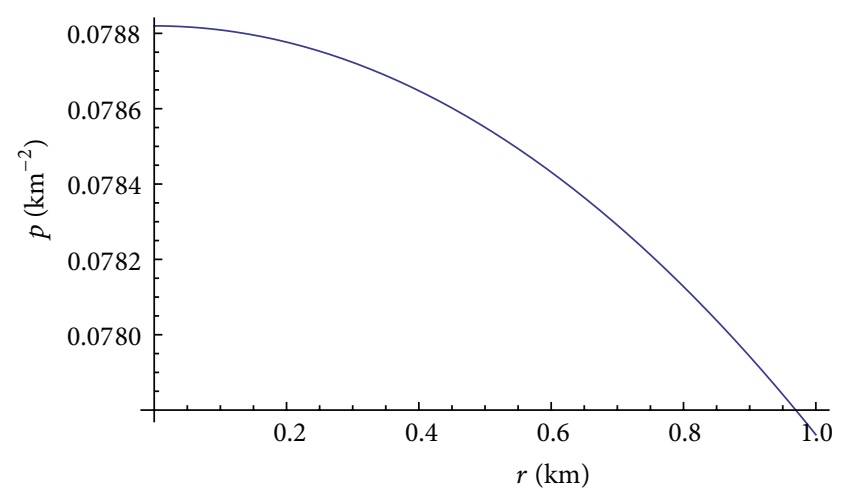

FIGURE 4: Matter pressure.

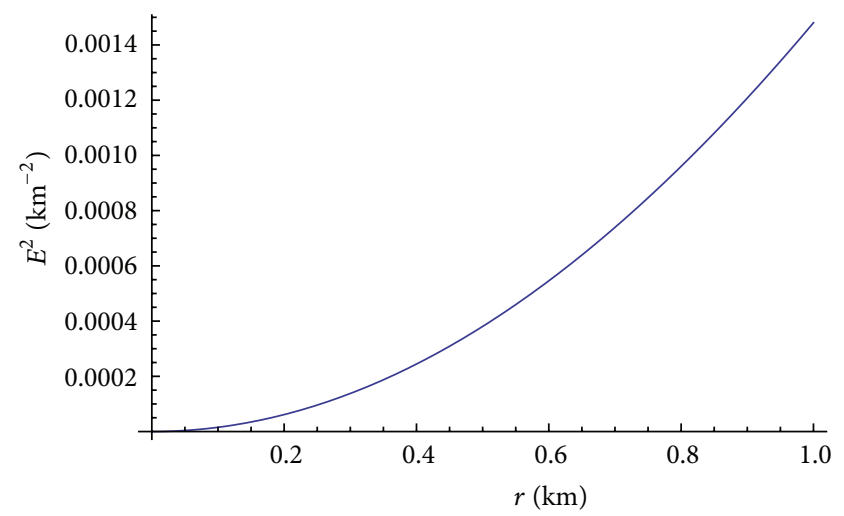

FIGURE 5: Electric field intensity.

similar to the value reported in the past [27]. However, for this particular example of parameter values considered in Table 1, the casuality condition is not satisfied as for the results obtained by Kumar and Gupta[23] and the argument given [23] for this scenario is notable.

\section{Conclusion}

New classes of solutions are generated to the Einstein-Maxwell system by assuming that the hypersurface $t=$ constant is spheroidal. For a particular choice of electric field intensity 
TABLE 1: $b$, radius of the star; $p(0)$, central pressure; $\rho(b)$, surface density; $\rho(0)$, central density; $q(b)$, surface charge.

\begin{tabular}{|c|c|c|c|c|c|c|c|}
\hline $\begin{array}{l}\text { Strange star } \\
\text { candidate }\end{array}$ & $R(\mathrm{~km})$ & $b(\mathrm{~km})$ & $p(0)\left(\mathrm{km}^{-2}\right)$ & $\rho(b)\left(\mathrm{km}^{-2}\right)$ & $\rho(0)\left(\mathrm{km}^{-2}\right)$ & $q(b)(\mathrm{km})$ & $\operatorname{Mass}\left(M_{\odot}\right)$ \\
\hline $\begin{array}{l}\text { SAX J1808.4-3658 } \\
\text { (SS1) }\end{array}$ & 8.672542 & 7.07 & 0.465 & 0.023 & 0.199 & 3.478 & 1.5295 \\
\hline $\begin{array}{l}\text { SAX J1808.4-3658 } \\
\text { (SS2) }\end{array}$ & 7.789341 & 6.35 & 0.576 & 0.016 & 0.247 & 3.883 & 1.3739 \\
\hline Her. X-1 & 9.445343 & 7.7 & 0.518 & 0.026 & 0.222 & 3.296 & 1.6658 \\
\hline $4 \mathrm{U} 1820-30$ & 12.266679 & 10 & 0.233 & 0.019 & 0.099 & 4.920 & 2.1633 \\
\hline
\end{tabular}

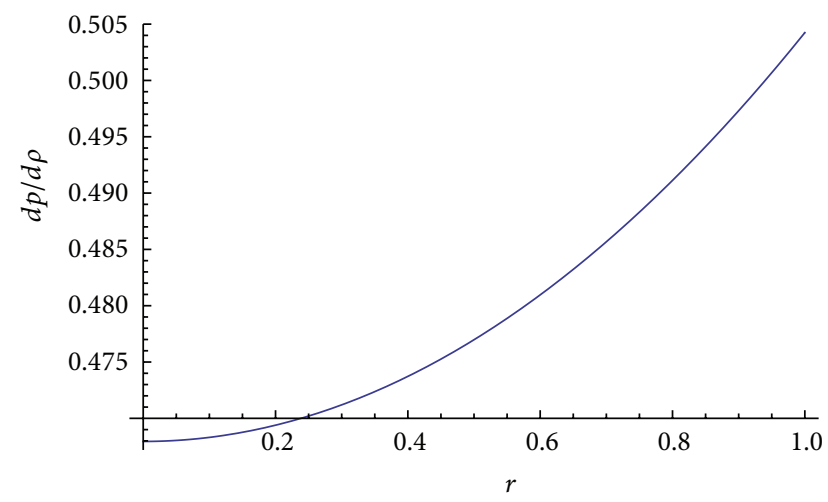

Figure 6: The derivative $d p / d \rho$.

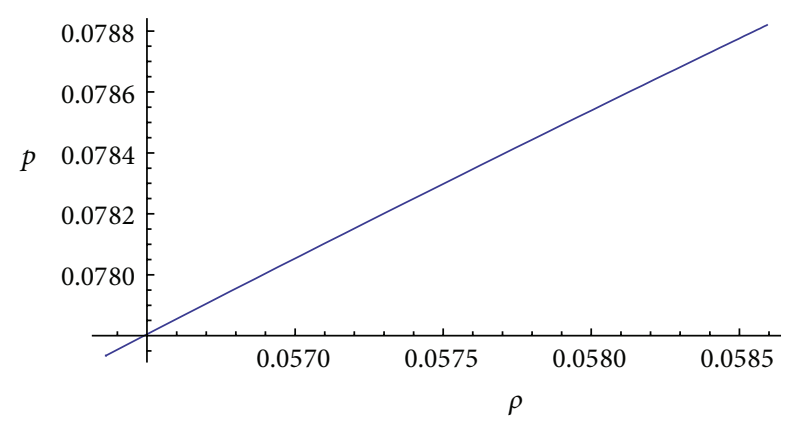

FIGURE 7: Pressure versus density.

$(\beta=-1)$, a class of solution is found for all positive spheroidal parameter values $K$. In general, a series solution is generated to the Einstein-Maxwell system and is demonstrated that two classes of solutions (40) and (42) can be extracted in terms of elementary functions. These class of solutions contain de Sitter model, Einstein universe, Tikekar superdense star [13] and Maharaj and Leach neutron star model [14] in the limit of vanishing electric field intensity. For particular parameter values, it is shown geometrically that the model satisfy the necessary physical requirements in the description of a compact object with isotropic matter distribution. Also, the mass values obtained are comparable with experimentally estimated values of realistic stars such as SAX J1808.43658(SS1), SAX J1808.4-3658(SS1), Her. X-1, and 4U 1820-30. We believe that the general class of exact solutions found in this paper may assist in more detailed studies of relativistic compact objects.

\section{References}

[1] W. B. Bonnor, "The mass of a static charged sphere," Zeitschrift für Physik, vol. 160, no. 1, pp. 59-65, 1960.

[2] B. V. Ivanov, "Static charged perfect fluid spheres in general relativity," Physical Review D, vol. 65, Article ID 104001, 17 pages, 2002.

[3] R. Sharma, S. Mukherjee, and S. D. Maharaj, "General solution for a class of static charged spheres," General Relativity and Gravitation, vol. 33, no. 6, pp. 999-1009, 2001.

[4] S. Thirukkanesh and S. D. Maharaj, "Charged relativistic spheres with generalized potentials," Mathematical Methods in the Applied Sciences, vol. 32, no. 6, pp. 684-701, 2009.

[5] Y. K. Gupta and S. K. Maurya, "A class of regular and well behaved charge analogue of Kuchowicz's relativistic superdense star model," Astrophysics and Space Science, vol. 332, no. 2, pp. 415-421, 2011.

[6] R. Sharma, S. Karmakar, and S. Mukherjee, "Maximum mass of a cold compact star," International Journal of Modern Physics D, vol. 15, pp. 405-418, 2006.

[7] R. Sharma and S. Mukherjee, "Compact stars: a core-envelope model," Modern Physics Letters A, vol. 17, p. 2535, 2002.

[8] R. Sharma and S. Mukherjee, "Her X-1: a quark diquark star?" Modern Physics Letters A, vol. 16, p. 1049, 2001.

[9] V. O. Thomas, B. S. Ratanpal, and P. C. Vinodkumar, "Coreenvelope models of superdense star with anisotropic envelope," International Journal of Modern Physics D, vol. 14, no. 1, pp. 8596, 2005.

[10] R. Tikekar and V. O. Thomas, "Relativistic fluid sphere on pseudo-spheroidal space-time," Pramana, vol. 50, no. 2, pp. 95103, 1998.

[11] B. C. Paul and R. Tikekar, "A core-envelope model of compact stars," Gravitation and Cosmology, vol. 11, pp. 244-248, 2005.

[12] P. C. Vaidya and R. Tikekar, "Exact relativistic model for a superdense star," Journal of Astrophysics and Astronomy, vol. 3, no. 3, pp. 325-334, 1982.

[13] R. Tikekar, "Exact model for a relativistic star," Journal of Mathematical Physics, vol. 31, no. 10, pp. 2454-2458, 1990.

[14] S. D. Maharaj and P. G. L. Leach, "Exact solutions for the Tikekar superdense star," Journal of Mathematical Physics, vol. 37, no. 1, pp. 430-437, 1996.

[15] K. Komathiraj and S. D. Maharaj, "Tikekar superdense stars in electric fields," Journal of Mathematical Physics, vol. 48, Article ID 042501, 12 pages, 2007.

[16] L. K. Patel and S. K. Koppar, "A charged analogue of the VaidyaTikekar solution," Australian Journal of Physics, vol. 40, pp. 441447, 1987. 
[17] R. Tikekar and G. P. Singh, "Interior reissner-nordstrom metric on spheroidal space-times," Gravitation and Cosmology, vol. 4, pp. 294-296, 1998.

[18] Y. K. Gupta and M. Kumar, "On the general solution for a class of charged fluid spheres," General Relativity and Gravitation, vol. 37, no. 1, pp. 233-236, 2005.

[19] Y. K. Gupta, Pratibha, and S. R. Kumar, "Some nonconformal accelerating perfect fluid plates of embedding class 1 using similarity transformations," International Journal of Modern Physics A, vol. 25, p. 1863, 2010.

[20] Y. K. Gupta, Pratibha, and J. Kumar, "A new class of charged analogues of Vaidya-Tikekar type super-dense star," Astrophysics and Space Science, vol. 333, no. 1, pp. 143-148, 2011.

[21] N. Bijalwan and Y. K. Gupta, "Closed form Vaidya-Tikekar type charged fluid spheres with pressure," Astrophysics and Space Science, vol. 334, pp. 293-299, 2011.

[22] N. Bijalwan, "Exact solutions: neutral and charged static perfect fluids with pressure," Astrophysics and Space Science, vol. 337, pp. 161-167, 2012.

[23] J. Kumar and Y. K. Gupta, "A class of new solutions of generalized charged analogues of Buchdahl's type super-dense star," Astrophysics and Space Science, vol. 345, pp. 331-337, 2013.

[24] X.-D. Li, I. Bombaci, M. Dey, J. Dey, and E. P. J. van den Heuvel, "Is SAX J1808.4-3658 a strange star?" Physical Review Letters, vol. 83, no. 19, pp. 3776-3779, 1999.

[25] M. Dey, I. Bombaci, J. Dey, S. Ray, and B. C. Samanta, "Strange stars with realistic quark vector interaction and phenomenological density-dependent scalar potential," Physics Letters B, vol. 438, no. 1-2, pp. 123-128, 1998.

[26] R. Sharma and S. D. Maharaj, "A class of relativistic stars with a linear equation of state," Monthly Notices of the Royal Astronomical Society, vol. 375, pp. 1265-1268, 2007.

[27] R. Tikekar and K. Jotania, "On relativistic models of strange stars," Pramana, vol. 68, no. 3, pp. 397-406, 2007.

[28] C. Alcock, E. Farhi, and A. Olinto, "Strange stars," The Astrophysical Journal, vol. 310, pp. 261-272, 1986. 

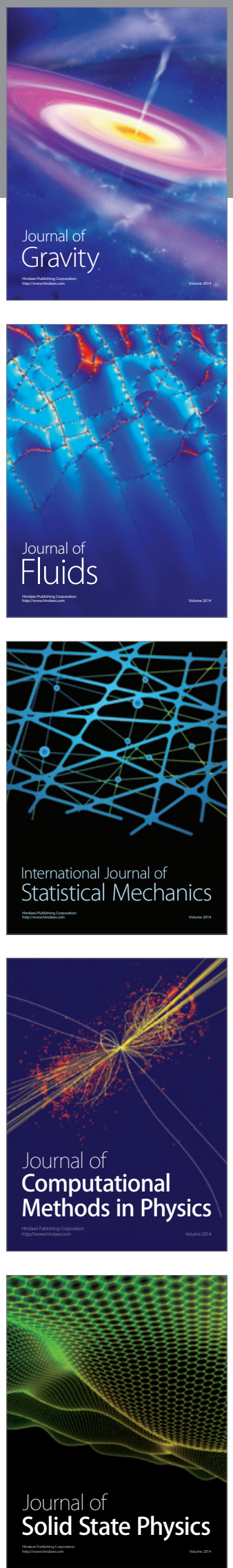

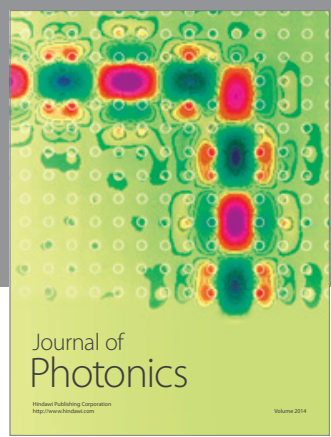

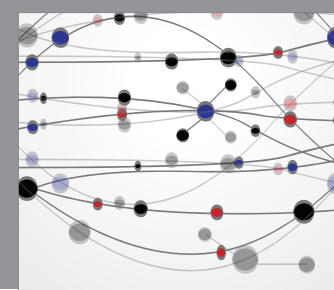

The Scientific World Journal

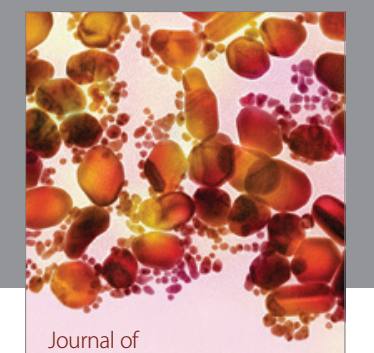

Soft Matter
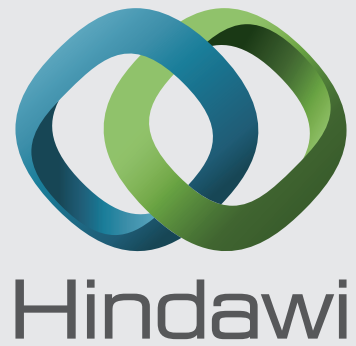

Submit your manuscripts at

http://www.hindawi.com
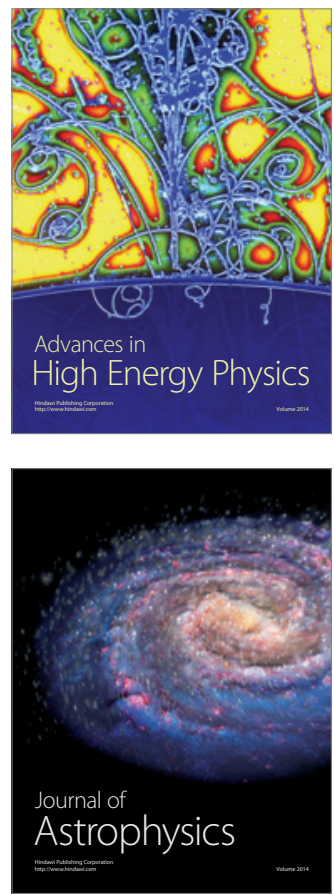
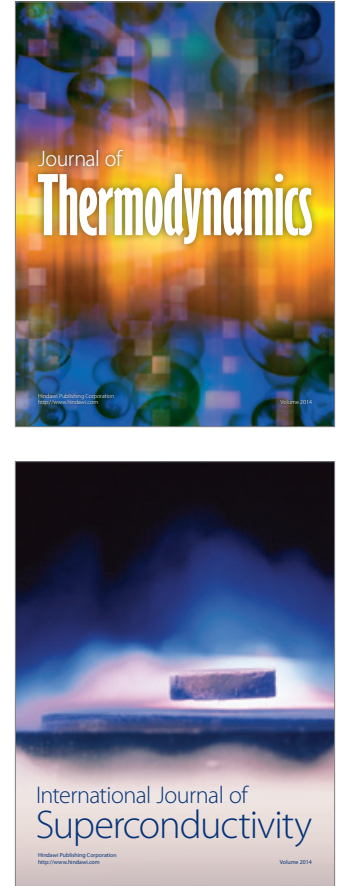
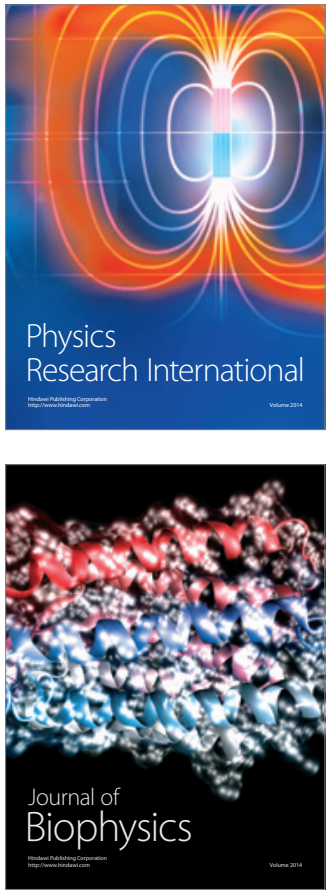
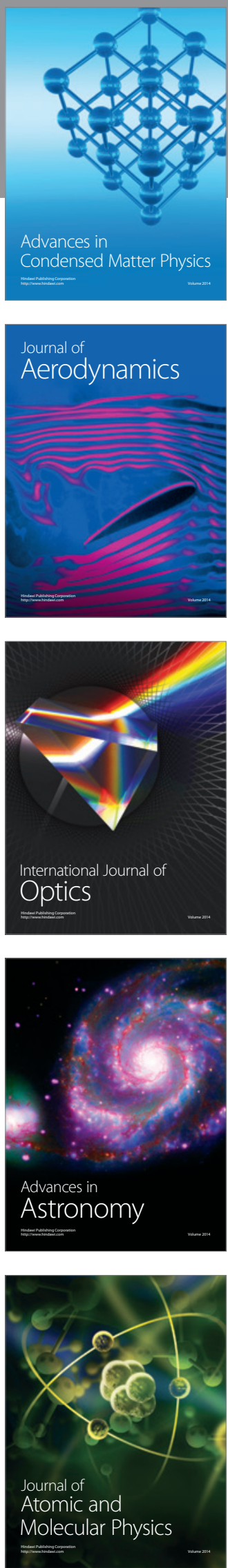